\title{
First Metatarsal Pronation Stratified by Tibial Sesamoid Position on Coronal Plane: A Study Based on Weight-Bearing Computed Tomography
}

\section{Zhi Wang}

Beijing Tongren Hospital

HE huijing

Chinese Academy of Medical Sciences and Peking Union Medical College Institute of Basic Medical

Sciences

\section{ZHANG Shu}

Beijing Tongren Hospital

\section{SUN Chao}

Beijing Tongren Hospital

\section{WANG Xianjun}

Beijing Tongren Hospital

\section{ZHANG Jianzhong}

Beijing Tongren Hospital

Zhang Mingzhu ( $\square$ michaelzhang120@hotmail.com )

Beijing Tongren Hospital, Capital Medical University https://orcid.org/0000-0002-6975-4965

\section{Research}

Keywords: Hallux Valgus, Weightbearing Computed tomography, Metatarsal Rotation

Posted Date: August 20th, 2021

DOl: https://doi.org/10.21203/rs.3.rs-770160/v1

License: (a) (1) This work is licensed under a Creative Commons Attribution 4.0 International License. Read Full License 


\section{Abstract}

Backgrounds: Few studies analyzed the coronal rotation of first metatarsal in hallux valgus patients with weightbearing computed tomography (WBCT). The purpose of this study is to investigate the association between hallux valgus parameters and first metatarsal head rotation in the coronal plane.

Methods: Retrospective review of full standing WBCT scans were taken by PedCAT. A group of 123 patients with 216 feet, that intermetatarsal angle $(\mathrm{IMA})>9$ degrees and hallux valgus angle (HVA) $>15$ degrees, were included. 27 feet of 17 female included in control group. Measurements of forefoot hallux valgus parameters included IMA, HVA, distal metatarsal abduction angle (DMAA), metatarsal adduction angle (MAA), first metatarsal coronal rotation angle (a-angle), tibial sesamoid position (TSP) on dorsoplantar view, and sesamoid grading in the coronal plane. The differences of HVA, IMA, MAA, DMAA and a-angle in control and study group were compared by Wilcoxon rank test and student $t$ test. Spearman correlation test was performed between a-angle and other parameters. General linear regression analysis was used to predict relationship parameters and a-angle.

Results: The mean a-angle in the control group was $11.33 \pm 6.16$ (range ,0-20) degrees, with the $95 \%$ confidence interval $(\mathrm{Cl})$ for the mean from 8.90 degrees to 13.77 degrees. The mean of a-angle in the study group was $23.75 \pm 6.32$ (range,9-39) degrees, with the $95 \% \mathrm{Cl}$ from 22.90 degrees to 24.59 degrees. In the hallux valgus group, there were positive correlations between a-angle and IMA (Spearman's correlation coefficient $\left.\left(r_{s}\right) 0.137, P=0.044\right)$, DMAA $\left(r_{s}=0.176, P=.010\right)$ and TSP $\left(r_{s}=0.218, P=0.001\right)$. General linear regression analysis showed that a-angle was correlated with TSP $(P<0.001)$. 208 hallux valgus feet $(96.3 \%)$ had more pronation than the control group, whereas 8 feet $(3.7 \%)$ had no or less pronation. Comparing the control and study subjects with the same TSP, means of a-angle showed significant difference, $\mathrm{P}=0.005$.

Conclusion: A large portion of hallux valgus patients had the first metatarsal pronation, which was positive correlated to TSP, IMA, and DMAA, and did not correlated to HVA and Smith grading. TSP is a useful predicting factor to a-angle changing.

Level of Evidence: Level $\otimes \bigotimes$ Retrospective Cohort study

\section{Introduction}

Hallux valgus is the most common deformity of the first metatarsophalangeal joint. It was reported that hallux and sesamoids rotate into pronation even in mild hallux valgus deformity. ${ }^{16,19}$ Recent reports showed that first metatarsal had rotation in the coronal plane, which was confirmed by simulating weightbearing CT and weightbearing $X$ ray. $3,12,13,14$ Kim reported a large sample of cases and found that first metatarsal rotation did not correlated with other measurement. ${ }^{12,13}$ But there were no standing weightbearing CT study of large sample investigation of hallux valgus yet. 
Our research focused on the relationship between first metatarsal coronal rotation and other hallux valgus measurements using standing WBCT. It had advantages, such as single scan procedure to obtain all radiographs, and more nature gesture during scanning. We focused on female only, since most patients were females. The aim of this study was therefore to retrospectively analyze the first metatarsal rotation in the coronal plane of hallux valgus. We hypothesized that the first metatarsal rotation in the coronal plane was an independent parameter.

\section{Methods}

This retrospective study and waiver of consent was approved by Medical Ethics Committee of our hospital. With a margin of error (MOE) of $\pm 2 \%$ and reported standard deviation 4.1 degrees and 6.0 degrees, ${ }^{12}$ the minimal sample size of control and study group would need to be 16 and 35 , respectively. Sample size of the control group and the study group were calculated by formula $n=z^{2} \times \sigma^{2} / M^{2} E^{2}(n$ : sample size; $z$ : found by using a z-score table; $\sigma$ : population standard deviation; MOE: margin of error). We added additional $20 \%$ to the minimum sample size, factoring in possible noncompliance rate, and finally targeted 20 and 42 cases.

The study cases were retrospectively reviewed from Oct 2017 to Dec 2018, which were standing WBCT records of our patients. The study group met the inclusion criteria as follows: (1) older than 18 years; (2) symptomatic hallux valgus deformity; (3) intermetatarsal angle (IMA) larger than 9 degrees, hallux valgus angle (HVA) larger than 15 degrees; and (4) preoperative first metatarsophalangeal joint total range of motion of more than 60 degrees. The exclusion criteria included the following: (1) previous foot trauma; (2) history of foot and ankle surgery; (3) hallux rigidus and severe first metatarsophalangeal joint arthritis with total range of motion smaller than 60 degrees; (4) rheumatoid arthritis, gouty arthritis or other arthritis; (5)midfoot or hindfoot deformity; and(6)neuromuscular disorder or muscular strength abnormalities. Finally, there were 216 feet of 123 female patients included in the hallux valgus group.

Retrospective review of WBCT records, which had no foot deformity and no lower extremity surgery or trauma history were selected and were included as candidates for the control group. All the feet which had IMA smaller than 9 degrees, and HVA smaller than 15 degrees were included. ${ }^{7,9}$ Then all cases filtered by the exclusion criteria. At last, 27 feet of 17 female included in control group.

One technician performed all WBCT scanning procedures. The PedCAT (CurveBeam, 2800 Bronze Drive, Suite 110, Hatfield PA 19440) weightbearing system was used to obtain all radiographic data. CubeVue software provided a reconstructed 3D view and slices in 3D coordinates. The Instant X (CubeVue software unit) was used as our plain film substitution. The measurements of radiographic parameters on the dorsoplantar view in the Instant X screenshot included IMA, HVA, distal metatarsal abduction angle (DMAA) and metatarsus adductus angle (MAA). The measurement method of first metatarsal axis used in our study was reported by Shima(Fig. 1A). ${ }^{17}$ The first metatarsal longitudinal axis was defined as the line connecting the center of the first metatarsal head to the center of the proximal articular surface. ${ }^{17}$ The Hardy-Clapham sesamoid classification on the dorsoplantar view was also recorded as tibial 
sesamoid position(TSP) scores in this study. ${ }^{7,12,20}$ First metatarsal coronal rotation angle was measured and recorded as a-angle reported by Kim (Fig. 1B). ${ }^{11,12}$ Sesamoid 4 position in the coronal plane was recorded as Smith grading. ${ }^{18,21}$ One doctor (first author) measured the parameters independently 3 times with a time interval of 4 weeks. After three measurements, the mean value was used as the data for statistical analysis.

\section{STATISTICAL METHOD}

Statistical analyses were performed by SPSS 22 (SPSS, Inc, an IBM Company, Chicago, IL). The difference of means of parameters in control and study group were compared by student $t$ test or Wilcoxon rank test according to whether data satisfied the Gaussian distribution. Spearman correlations between $\alpha$-angle and other angular parameters were processed. General linear regression analysis was used to predict relationship between angular parameters and a-angle. A $P$ value less than 0.05 (twosided) was considered statistically significant.

\section{Results}

Subjects in both groups exceeded the minimal sample size estimation. There were all female feet, there was no difference in the means of age for the two groups. Angles between the subjects and controls were compared (Table 1). Differences between IMA, HVA, DMAA, TSP, MAA, Smith grading and a-angle were statistically significant, all $\mathrm{P}<.001$. 
Table 1

Comparison of age, IMA, HVA, a-angle, distribution of TSP, TSCG between groups

\begin{tabular}{|c|c|c|c|c|}
\hline Variables & $\begin{array}{l}\text { Hallux valgus } \\
(n=119)\end{array}$ & $\begin{array}{l}\text { Control } \\
(n=27)\end{array}$ & $\chi^{2}, z$ & $P$ \\
\hline Age (Years old, Median, P25, P75) & $56(47,63)$ & $50(46,61)$ & -1.253 & 0.210 \\
\hline IMA $\left({ }^{\circ}\right.$, Median, P25, P75) & $14(12,16)$ & $8(7,9)$ & -8.127 & $<0.001$ \\
\hline HVA $\left({ }^{\circ}\right.$, Median, P25, P75) & $31(25,38)$ & $11(10,13)$ & -8.071 & $<0.001$ \\
\hline $\operatorname{DMAA}\left({ }^{\circ}\right.$, Median, $\left.\mathrm{P} 25, \mathrm{P} 75\right)$ & $19(15,25)$ & $5(2,8)$ & -7.363 & $<0.001$ \\
\hline $\operatorname{MAA}\left({ }^{\circ}\right.$, Median, P25, P75) & $15(12,19)$ & $12(10,17)$ & -2.407 & 0.016 \\
\hline a-angle (Median, P25, P75) & $24(19,29)$ & $13(9,18)$ & -6.764 & $<0.001$ \\
\hline $\operatorname{TSP}(n, \%)$ & $0(0)$ & $1(3.70)$ & 97.535 & $<0.001$ \\
\hline 1 & $0(0)$ & 9 (33.33) & & \\
\hline 2 & $6(5.04 \%)$ & $13(48.15)$ & & \\
\hline 3 & $16(13.45 \%)$ & $4(14.81)$ & & \\
\hline 4 & $23(19.33 \%)$ & $0(0)$ & & \\
\hline 5 & $44(36.97 \%)$ & $0(0)$ & & \\
\hline 6 & $30(25.21 \%)$ & $0(0)$ & & \\
\hline \multicolumn{5}{|l|}{7} \\
\hline $\operatorname{TSCG}(n, \%)$ & $21(17.65 \%)$ & $27(100)$ & 67.632 & $<0.001$ \\
\hline 0 & 31 (26.05\%) & $0(0)$ & & \\
\hline 1 & 38 (31.93\%) & $0(0)$ & & \\
\hline 2 & $29(24.37 \%)$ & $0(0)$ & & \\
\hline 3 & & & & \\
\hline
\end{tabular}

The mean a-angle in the control group was $11.33 \pm 6.16$ (range, 0-20) degrees, with the $95 \%$ confidence interval $(\mathrm{Cl})$ for the mean from 8.90 degrees to 13.77 degrees. The mean of a-angle in the study group was $23.75 \pm 6.32$ (range, $9-39$ ) degrees, with the $95 \% \mathrm{Cl}$ from 22.90 degrees to 24.59 degrees. All hallux valgus feet had the first metatarsal rotating in pronation, and there was no supination.

Compared with the control group, the hallux valgus group had 12.42 degrees greater pronation on average. Figure 2 is the distribution of the a-angle in all cases. 
In the hallux valgus group, there were positive correlations between a-angle and IMA (Spearman's correlation coefficient $\left.\left(r_{s}\right) 0.137, P=.044\right)$, DMAA $\left(r_{s}=.176, P=.010\right)$ and TSP $\left(r_{s}=.218, P=.001\right)$. In the general linear regression model of the study group, a-angle was associated with TSP with statistical significance, $\mathrm{P}<.001$.

Case number distribution by sesamoid grading was exhibited on Table 2 and Table 3. In the study group, increasing of a-angle could be observed in different TSP group. There were only 8 feet $(8 / 216,3.7 \%)$ had a-angle smaller than 13.77 degrees. 24 feet $(24 / 216,11.11 \%)$ did not have subluxation of sesamoids on coronal plane.

Table 2

Spearman Correlation versus $a$-angle in the study group

\begin{tabular}{|lll|}
\hline Variables & Spearman coefficient versus a-angle $(\mathbf{n}=119)$ & P-value \\
\hline age & 0.015 & 0.873 \\
\hline IMA & 0.139 & 0.132 \\
\hline HVA & 0.001 & 0.994 \\
\hline DMAA & 0.096 & 0.299 \\
\hline MAA & -0.147 & 0.112 \\
\hline TSP & 0.206 & $0.025^{\star}$ \\
\hline TSCG & -0.098 & 0.289 \\
\hline $\begin{array}{l}\text { Abbreviations: IMA, Intermetatarsal angle; HVA, Hallux valgus angle; DMAA, Distal metatarsal articular } \\
\text { angle; MAA, metatarsal adduction angle; TSP, Tibial sesamoid position; TSCG, tibial sesamoid coronal } \\
\text { grading. }\end{array}$ & & \\
\hline
\end{tabular}


Table 3

The association between factors and a-angle yielded by the general linear regression models, and stratification analyses based on TSCG classification (limited in the Hallux valgus group)

\begin{tabular}{|c|c|c|c|c|c|c|c|c|}
\hline \multirow[t]{2}{*}{ Variables } & \multicolumn{4}{|c|}{ Hallux valgus $(n=119)$} & \multicolumn{4}{|c|}{ Control $(n=27)$} \\
\hline & $\beta^{\#}$ & $95 \% \mathrm{Cl}$ & & $p$ & $\beta^{\#}$ & $95 \% \mathrm{Cl}$ & & $p$ \\
\hline HVA & -0.0361 & -0.2044 & 0.1323 & 0.6746 & 0.369 & -0.5426 & 1.2807 & 0.4275 \\
\hline Age & -0.0054 & -0.0915 & 0.0808 & 0.9028 & 0.0559 & -0.146 & 0.2579 & 0.5873 \\
\hline IMA & 0.0061 & -0.4844 & 0.4967 & 0.9804 & 1.1296 & -1.1871 & 3.4464 & 0.3392 \\
\hline DMAA & 0.05 & -0.1321 & 0.2321 & 0.5902 & -0.1302 & -0.6398 & 0.3794 & 0.6165 \\
\hline MAA & -0.1984 & -0.4116 & 0.0147 & 0.068 & -0.4592 & -0.9842 & 0.0659 & 0.0865 \\
\hline TSP & 3.2038 & 1.7981 & 4.6096 & $<.0001$ & 3.7106 & 0.8983 & 6.5229 & 0.0097 \\
\hline \multicolumn{9}{|l|}{ TSCG } \\
\hline 0 & 8.5046 & 4.1395 & 12.8698 & 0.0001 & & & & \\
\hline 1 & 4.1516 & 0.7365 & 7.5667 & 0.0172 & & & & \\
\hline 2 & 1.9881 & -0.9675 & 4.9437 & 0.1874 & & & & \\
\hline 3 (ref) & 0 & NA & NA & NA & & & & \\
\hline
\end{tabular}

Table 4

TSP and a-angle correlation based on TSCG categories.

\begin{tabular}{|llllll|}
\hline TSCG strata & $\mathbf{n}$ & \multicolumn{3}{l}{ TSP } & \\
\cline { 3 - 6 } & & $\boldsymbol{\beta}^{\#}$ & $95 \%$ Cl of $\boldsymbol{\beta}$ & \\
\hline 0 & 21 & 5.7047 & 3.8418 & 7.5675 & $<.0001$ \\
\hline 1 & 31 & 3.3079 & 0.9537 & 5.6621 & 0.0059 \\
\hline 2 & 38 & 5.9009 & 3.2376 & 8.5642 & $<.0001$ \\
\hline 3 & 29 & -3.8244 & -7.2262 & -0.4226 & 0.0276 \\
\hline $\begin{array}{l}\text { Abbreviations: TSCG, tibial sesamoid coronal grading; TSP, Tibial sesamoid position; } 95 \% \mathrm{Cl}, 95 \% \\
\text { confidence interval. }\end{array}$
\end{tabular}


Table 5

Means of variables and standard deviations in different TSCG strata.

\begin{tabular}{|c|c|c|c|c|c|c|c|}
\hline $\begin{array}{l}\text { TSCG } \\
\text { Strata }\end{array}$ & $n$ & $\begin{array}{l}\text { Mean } \\
\text { of IMA } \\
\text { (degree, } \\
\text { SD) }\end{array}$ & $\begin{array}{l}\text { Mean } \\
\text { of HVA } \\
\text { (degree, } \\
\text { SD) }\end{array}$ & $\begin{array}{l}\text { Mean of } \\
\text { DMAA } \\
\text { (degree, } \\
\text { SD) }\end{array}$ & $\begin{array}{l}\text { Mean } \\
\text { of MAA } \\
\text { (degree, } \\
\text { SD) }\end{array}$ & $\begin{array}{l}\text { Mean of } \\
\text { a-angle } \\
\text { (degree, } \\
\text { SD) }\end{array}$ & $\begin{array}{l}\text { The adjusted mean of } a- \\
\text { angle (degree) }\end{array}$ \\
\hline 0 & 21 & $\begin{array}{l}12.38 \\
2.06\end{array}$ & $\begin{array}{l}24.86 \\
6.48\end{array}$ & $\begin{array}{l}14.05 \\
6.29\end{array}$ & $\begin{array}{l}13.19 \\
4.24\end{array}$ & $\begin{array}{l}25.81 \\
6.52\end{array}$ & 28.29 \\
\hline 1 & 31 & $\begin{array}{l}13.35 \\
2.04\end{array}$ & $\begin{array}{l}28.00 \\
6.90\end{array}$ & $\begin{array}{l}18.32 \\
5.28\end{array}$ & $\begin{array}{l}\text { 16.00 } \\
5.97\end{array}$ & $\begin{array}{l}24.55 \\
5.74\end{array}$ & 23.40 \\
\hline 2 & 38 & $\begin{array}{l}14.97 \\
3.16\end{array}$ & $\begin{array}{l}32.47 \\
7.21\end{array}$ & $\begin{array}{l}21.71 \\
6.91\end{array}$ & $\begin{array}{l}15.13 \\
5.90\end{array}$ & $\begin{array}{l}24.87 \\
7.47\end{array}$ & 21.73 \\
\hline 3 & 29 & $\begin{array}{l}15.10 \\
2.73\end{array}$ & $\begin{array}{l}39.48 \\
8.77\end{array}$ & $\begin{array}{l}23.10 \\
7.44\end{array}$ & $\begin{array}{l}17.34 \\
4.27\end{array}$ & $\begin{array}{l}23.21 \\
5.75\end{array}$ & 19.65 \\
\hline$P$ & & 0.001 & $<0.001$ & $<0.001$ & 0.025 & 0.670 & $\mathrm{~N} / \mathrm{A}$ \\
\hline
\end{tabular}

There were 16 feet in control group and 7 feet in study group classified as TSP grade 3. Means of a-angle were $9.81 \pm 6.21$ degrees and $18.71 \pm 5.19$ degrees respectively. Wilcoxon rank test show significant difference of a-angle between subgroup of cases, $P=.005$.

\section{Discussion}

The first metatarsal rotation in the coronal plane is critical in hallux valgus. Direct measurement of first metatarsal rotation and sesamoid subluxation is difficult. This study only focused on female patient, excluded gender difference from male patients. It revealed that hallux valgus deformity had more first metatarsal coronal rotation, and the a-angle correlation to IMA, DMAA, TSP.

The a-angle in control group was important to define abnormality. As adequate sample of control included, the average a-angle of 11.33 degrees was calculated. The average a-angle of the study group was 23.75 degrees, which was 12.42 degrees larger than the control group. All feet in study group had first metatarsal rotating into pronation, and there was no supination. This result was comparable to Kim and Mortier's report. ${ }^{12,13}$ However, the mean of a-angle was different with their reports. Kim reported that the $a$-angle in his control group was $13.8 \pm 4.1$ degrees, but in our study, this was $21.9 \pm 6.0$ degrees. ${ }^{12}$ There are several reasons of this difference. First, simulating WBCT provided pressure on supine with a compressing machine which was not normal stress to foot. Second, the control group had larger sample size and were comparable with the study group with age, therefore guaranteed enough statistical power. 
Third, weightbearing X-ray and simulating WBCT were taken separately in Kim's study. In our study, all radiographs acquired in single scanning.

Mortier et al. used weightbearing tangential radiographs to measure the first metatarsal pronation and reported a mean pronation value of $12.7 \pm 7.7$ degrees. ${ }^{13}$ The measurement of rotation on a plane radiograph may be influenced by a dorsiflexed first metatarsophalangeal joint. Collan et al. utilized WBCT to study the rotation in hallux valgus patients but showed no difference of coronal rotation between hallux valgus feet and normal feet. ${ }^{3}$ In their study, the WBCT only scanned one side at a time, not real physiological standing status. And their study had a small sample, which included 10 and 19 feet in the control and hallux valgus groups. So, our study was closer to the real physical condition.

Using the upper boundary of the $95 \% \mathrm{Cl}$ of the control group as boundary, there were 208 feet $(96.3 \%)$ of the study group had excessive first metatarsal pronation. This percentage was largest compare to other reports. Kim reported $87.3 \%$ of hallux valgus cases had first metatarsal pronation more than the upper bounder of $95 \% \mathrm{Cl}$ of control. ${ }^{12}$ And Dayton reported $81 \%$ of his cases had first metatarsal pronation. ${ }^{5}$

Most of patient had sesamoid subluxation, $88.9 \%$ of hallux valgus patients had subluxation of sesamoids, and on average had a TSP grade 5.7 and Smith grade 1.79 in the coronal plane. TSP grading distribution showed that 32 feet $(32 / 216,14.81 \%)$ had tibial sesamoid position smaller than grade five. in the coronal plane, only 24 feet $(24 / 216,11.11 \%)$ had no subluxation of sesamoids, as Smith grade zero, in which 23 feet (10.6\%) defined as pseudo-subluxation.

It was supposed that pseudo-subluxation hallux valgus feet will not require distal soft tissue release. But only $11.11 \%$ of cases were pseudo-subluxation. Smith and colleagues reported an anatomic triplane hallux valgus classification. ${ }^{8,18}$ Accordingly, in our study group, $11.11 \%$ of cases were Class 1 or Class $2 \mathrm{~A}$, and others were classified as Class $2 \mathrm{~B}$ and Class 3 . Average age was older, and IMA were relatively larger than. Only Most patients required distal soft tissue release. And age affects the sesamoids subluxation on coronal plane. ${ }^{10}$ Dayton and colleagues reported in their study, average age 32.42 years, ${ }^{4}$ that rotational correction without distal soft release reduce tibial sesamoid into its position. ${ }^{4,5}$

Rotational correction required on average was 22.1 degrees. ${ }^{5}$ They found that greater preoperative TSP scores were associated with greater intraoperative varus rotation required. ${ }^{5}$ In another case series with 21 feet, they reported that $81 \%(17 / 21)$ of cases had metatarsal pronation preoperatively. ${ }^{4}$

IMA, DMAA, and TSP had positive correlation to a-angle. But HVA and Smith grading had no correlation to a-angle. This was the difference between standing WBCT and simulating WBCT, or plane radiograph measurement. Kim reported that the rotation had no correlation among the parameters, such as IMA, HVA. Thus, first metatarsal pronation was independent to other hallux valgus changes. ${ }^{12}$ Motier also reported that first and second metatarsal angle had no correlation to radiographic pronation. ${ }^{13}$ 
IMA and TSP could be used as predictors of a-angle. But linear regression model showed that only TSP was a statistically predictable factor. Tibial sesamoid position(TSP) was used to evaluate the deformity of hallux valgus. ${ }^{6,15,20}$ Given that TSP was related to hallux valgus recurrence and failed correction, 1,15 TSP also related to the rotation of first metatarsal. ${ }^{1,11,15}$ Our study indicated the association between TSP and $a$-angle in hallux valgus group. TSP is very useful to predict coronal rotation of first metatarsal. In Table 3, a-angle increased along with TSP increased. This is the first time quantifying the changing of coronal rotation with TSP. Major difference was occurred even when TSP was relatively small.

Statistically, a-angle was different as the control and study group had same TSP grading 3 . This phenomenon may imply that first metatarsal rotation occurs in mild hallux valgus. In the study group, TSP grading increased in different subgroups but clinically minor in practice.

This study has several limitations. This study was a retrospective study of female hallux valgus patients. All parameters were measured by one doctor, however measurement of DMAA may be less variable. ${ }^{2}$ Furthermore, our measurements were carried from the 2-D definition. The first metatarsal pronation is a 3D rotation and mix with torsion of first metatarsal shaft. ${ }^{14}$ The a-angle maybe a primitive way to understand the complexity of the deformity and real 3-D measurements could reveal more details.

\section{Conclusion}

A large portion of hallux valgus patients had the first metatarsal pronation, which was positive correlated to TSP, IMA, and DMAA, and did not correlated to HVA and Smith grading. TSP is a useful predicting factor to a-angle changing. However, rotation may change little in different TSP subgroups of hallux valgus patients.

\section{Abbreviations}

WBCT weight-bearing computed tomography

IMA Intermetatarsal angle

HVA hallux valgus angle

DMAA distal metatarsal articular angle

MAA metatarsal adduction angle

TSP tibial sesamoid position

TSCG tibial sesamoid coronal grading

GLMs General linear regression models

$\mathrm{Cl}$ confidence interval 
AP anteroposterior

ROM range of motion

ICC intra-class correlation coefficient

\section{Declarations}

\section{Ethics approval and consent to participate}

This study was approved by Beijing Tongren Hospital Ethics Committee and is compliant with the Health Insurance Portability and Accountability Act (HIPAA) and the Declaration of Helsinki Informed consent was signed by all participants to be included in the study.

\section{Consent for publication}

All the personal data in this publication has consent by them to publish.

Availability of data and material

\section{Availability of data and materials}

Data sharing not applicable to this article as no data-sets were generated or analyzed during the current study

\section{Competing interests}

The authors declare that they have no competing interests

\section{Authors' contributions}

WZ and ZJ participated in the design of the study. WZ and SC developed the methods and measurement setup. WZ and QF organised the measurements, recruited the participants, collected data. $\mathrm{HH}$ and ZS performed the statistical analysis. WF and WX helped in data collection, interpretation of data. WZ and ZM conceived of the study, and participated in its design and coordination and helped to draft the manuscript. All authors read and approved the final manuscript.

\section{Acknowledgements}

Not applicable.

Funding: This study was supported by the National Key Research and Development Program of China (No.2017YFC0108103)

\section{Figures}



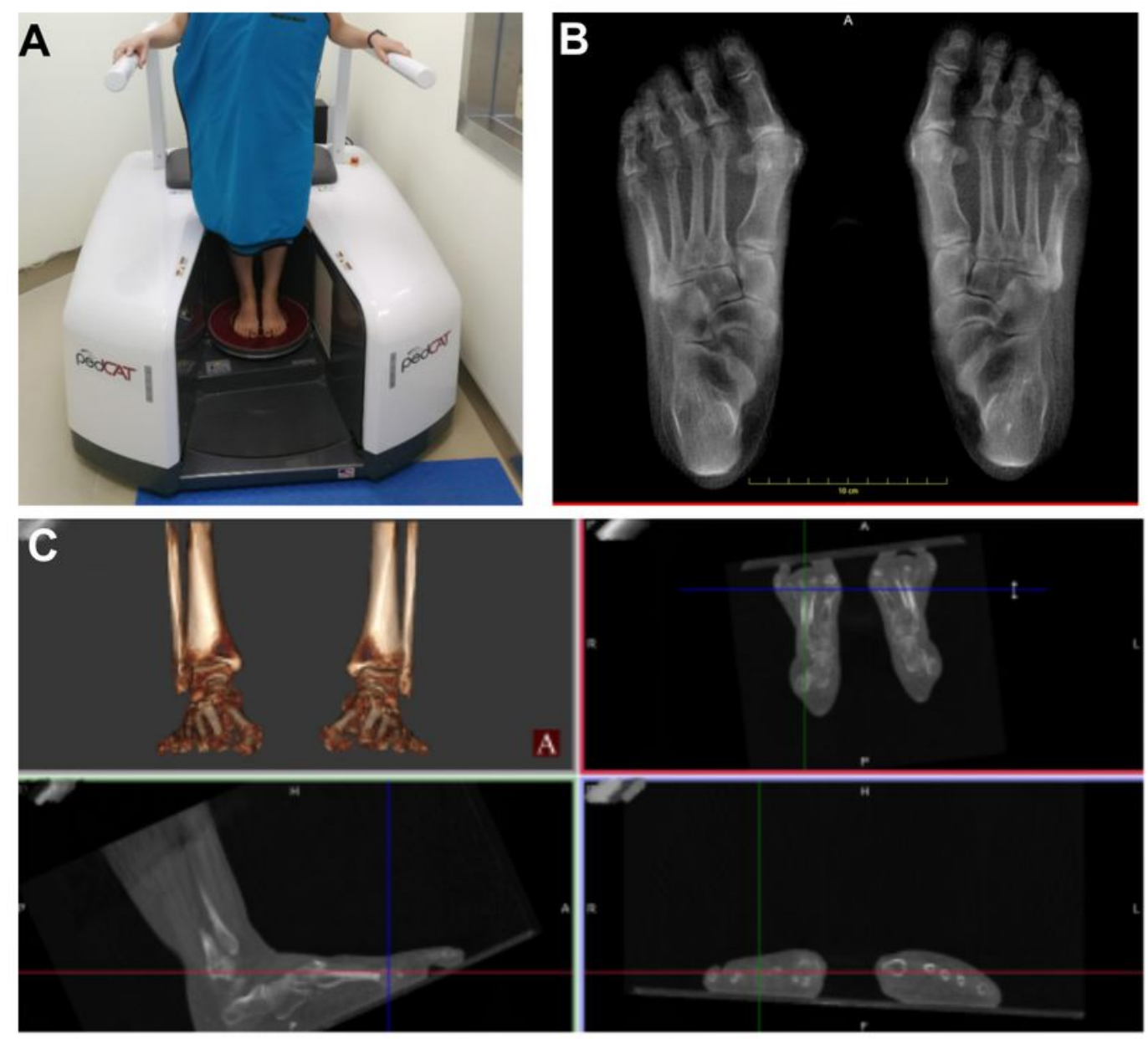

\section{D}

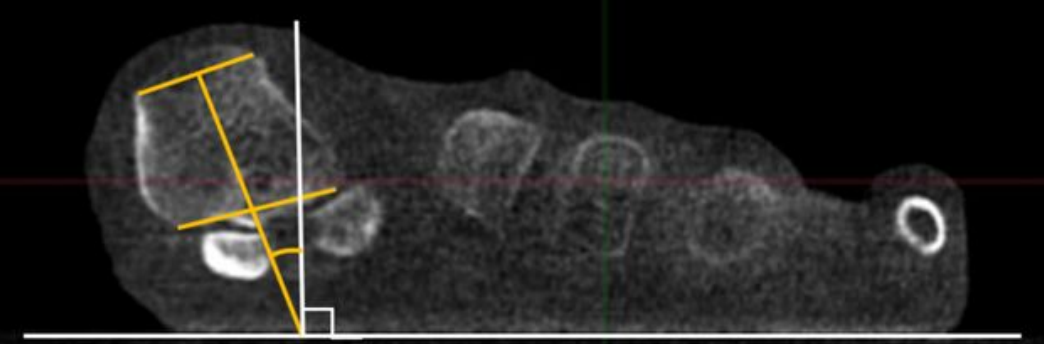

$\alpha$-angle

\section{Figure 1}

A The measurement of angles was assisted by software. And first metatarsal axis defined as Shima reported. B The axis adjustment was applied for all records. The first step was adjusting slices parallel to third metatarsal on the sagittal plane and frontal plane. Found sesamoids center and first metatarsal on coronal plane. This coronal image saved into the archives. 


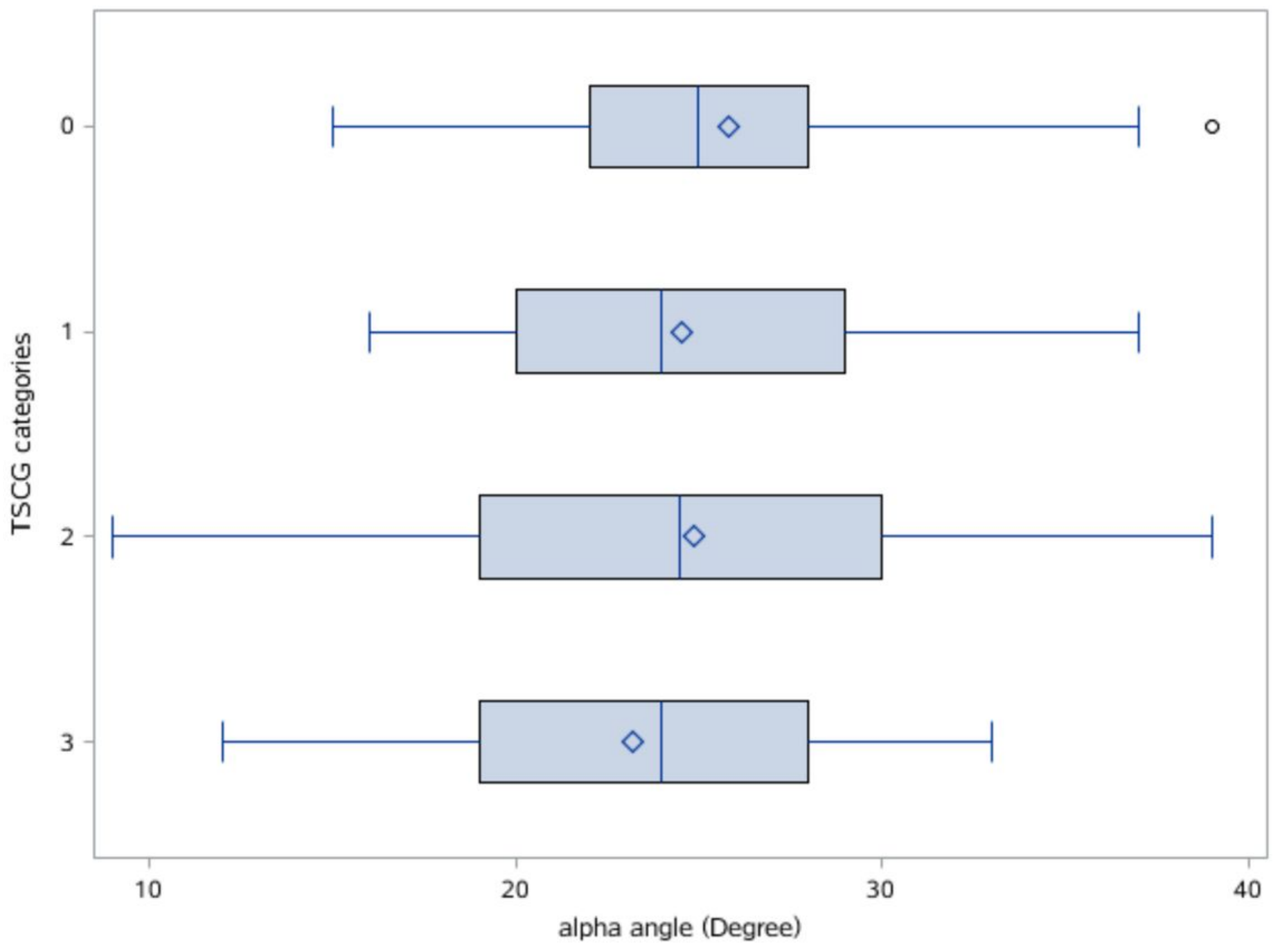

Figure 2

Distribution of a-angle of each subject along with the IMA. The a-angle and IMA had a positive correlation with statistical significance, $P=.044$. 